ISSN: 2174-5609

DOI. http://dx.doi.org/10.14198/INTURI2016.12.05

TJT Investigaciones

$\Delta$ teruncester TURISTICAS

\title{
Crecimiento y planeación urbana en Acapulco, Cancún y Puerto Vallarta (México)
}

\author{
Erika Patricia Cárdenas Gómez \\ El colegio de Jalisco, México \\ erika.cardenas@coljal.edu.mx
}

\section{RESUMEN}

El presente artículo tiene como objetivo dar cuenta del crecimiento urbano que han registrado tres ciudades mexicanas: Acapulco, Guerrero; Cancún, Quintana Roo y Puerto Vallarta, Jalisco. Así como establecer el vínculo que existe entre el crecimiento urbano y demográfico con el desarrollo de la actividad turística en dichos asentamientos urbanos. La importancia de analizar las tres ciudades seleccionadas obedece a que fue apenas en el siglo XX cuando cobraron gran relevancia. Aunque cabe enfatizar que su proceso de gestación no ha sido en los mismos años ni en las mismas condiciones. Pero aun es conveniente conocer ¿cuántas hectáreas se han urbanizado?, y ¿cómo es su planeación urbana?, entre otras interrogantes.

Cabe advertir que dicha temática ya ha sido planteada por varios autores aunque de manera particular, el aporte del presente artículo radica en que presenta los tres casos de estudios y que los datos están actualizados. Asimismo, en este trabajo también se mencionan los mecanismos que se han instrumentado para su planeación urbana.

Palabras clave: Crecimiento urbano, ordenamiento urbano, turismo, Acapulco, Cancún, Puerto Vallarta 


\title{
The Smart Tourism Destination and the Territorial Intelligence: problems and opportunities
}

\begin{abstract}
The following article has the objective of accounting for the urban growth registered in three Mexican cities: Acapulco, Guerrero; Cancún, Quintana Roo and Puerto Vallarta, Jalisco. As well as establishing the bond that there is between the urban and demographic growth with the development of touristic activity in the aforementioned urban settlements. The importance of analyzing the three selected cities is that it was just in the XX century when they took great relevance. Even though it is worth mentioning that their gestation process has not been in the same years nor conditions. However, it is convenient to find out among other questions: How many hectares have been urbanized? And, how is their urban planning?

It is convenient to say that several authors have questioned such topics in a particular way. The present article contributes in the aspect that it shows the three case studies and that the data is updated. Likewise, in this paper, the mechanisms that have been implemented for their urban planning are mentioned.
\end{abstract}

Keywords: Urban growth, urban planning, tourism, Acapulco, Cancun, Puerto Vallarta

\section{INTRODUCCIÓN}

Por principio de cuenta se debe enfatizar que con la Revolución Industrial el concepto de ciudad se consagró como la ganadora en la atracción de habitantes debido a que el morar en ellas trajo algunos beneficios, pues en ésta se concentra las industrias, las instituciones educativas y de salud, así como los espacios de entretenimiento. Aunque, cabe indicar que en la misma también prevalecen algunos problemas de desempleo, pobreza, inseguridad pública, insuficiencia del transporte público, deficiencias en la planeación urbana, entre otros (Garza y Sands, 2003, p. 15).

Ahora bien, dichas problemáticas se agudizarán aún más en las ciudades latinoamericanas, pues como lo señala Carrión éstas albergan ciertas peculiaridades, tales como: a) una urbanización sustentada en el incremento acelerado de la migración ruralurbana y b) el desarrollo urbano dual venido de los asentamientos humanos irregulares, informales e ilegales (2013, p. 23).

Pero habría que agregar que dichas particularidades serán más evidentes en las ciudades fronterizas y en las turísticas. Por ejemplo, en éstas últimas hay distinciones en la forma de ocupar y vivir el espacio entre los turistas, la población local y los migrantes pobres. Ello se debe a que la política urbana ha favorecido en gran medida el espacio que ocupa el visitante, al mismo tiempo relega los espacios de convivencia de la población nativa. Además, de que muchas de las veces éstos resultan ser menos atractivos (Bouvet citado por Castillo y Villar, 2011, p. 89).

Ahora bien, se debe enfatizar que por varios siglos las ciudades de litoral en México se encontraron aisladas debido a factores geográficos (Olveda, 2011). Aunque por esta 
misma situación conservaron sus riquezas naturales, las cuales fueron altamente cotizadas cuando se impulsó el turismo de masas, después de la Segunda Guerra Mundial. Pero para ello, el gobierno federal tuvo que jugar un papel importante. Al respecto Jiménez menciona que éste se encargó de la construcción de aeropuertos, carreteras, marinas, introducción de agua potable, energía eléctrica, urbanización y servicios turísticos (1992, p. 84), así como del financiamiento de hoteles (García, 1979; Ramírez, 1986; Oehmichen, 2013).

El primer destino de sol y playa que apoyó el gobierno federal fue Acapulco, le siguió Puerto Vallarta y en una política de corte "explícita" a Cancún (Hiernaux y Torres, 2008, p. 112). Por esta intervención las ciudades de litoral en México se clasifican, en dos grandes grupos: las tradicionales y los Centros Integralmente Planeados, (CIPS). Las primeras tienen su origen muy independiente de la actividad turística, (puede ser que en un inicio se hayan desempeñado como puertos), pero una vez que ésta se impulsa logran un rápido crecimiento económico, demográfico y urbano, como es el caso de Acapulco. Y los CIPS, los cuales son planeados y construidos por el Estado. ${ }^{1}$

Se debe destacar que para la construcción de los CIPS se canalizaron la mayor cantidad de recursos que el país destinó al rubro de turismo (Jiménez, 1992, p. 84). Aunque el financiamiento provino de préstamos internacionales (García, 1979; Hiernaux, 2010). El ejemplo más exitoso ha sido Cancún. Así pues, con estas medidas y otras más el gobierno ha buscado afanosamente atraer al turista internacional y de altos ingresos. Ello con la finalidad de recuperar la inversión, ejercida (Bringas, 1999, p. 7).

Una las razones de construir los CIPS obedece a poblar y crear polos de desarrollo en el litoral, el cual es poco más de 11000 kilómetros repartidos entre el Océano Pacífico y el Atlántico (Bringas, 1999, p. 5). Aunque, esta medida se inscribe también dentro de las corrientes de pensamiento económico internacional, pero su implementación en México, algunos autores la califican, como la "construcción de polos de subdesarrollo" (George, citado por García, 1979, p. 103). Ello se observará de manera tácita con el caso de Cancún.

Independientemente de su proceso de gestación, las ciudades turísticas de sol y playa se caracterizan por su forma de tipo lineal. Además, en su composición está presente la dualidad, pues cuentan con una parte, que se le denomina, la "fachada" y es la que se encuentra paralela a la línea de la playa (en ésta se localizan los hoteles, condominios, resorts, viviendas de segundas residencias, etcétera). La otra parte es la "trasera" y da la espalda al mar. En ella es donde viven los nativos (Díaz, 2013, p. 45). Sin embargo, es importante advertir que la parte trasera no es un territorio homogéneo debido a que al interior guarda sus propias diferencias, al dar cabida a la población migrante de bajos recursos económicos que anda en mejores oportunidades de vida. Por lo anterior y por otras razones se ha dado paso a la fuerte segregación socioespacial que se vive en estas urbes, a pesar de que la ciudad haya sido planeada (García, 1979).

De igual manera, se deben destacar los mecanismos que se han puesto en marcha para apropiarse de terrenos ejidales e impulsar los desarrollos turísticos. Éstos van desde

\footnotetext{
${ }^{1}$ Aunque cabe advertir que también existen casos donde las ciudades no entran dentro de esta tipificación, como es el caso de Puerto Vallarta que se encuentra en una fase intermedia entre ciudad tradicional y un CIP debido a que con ella se marca el inicio al apoyo brindado por parte de las instituciones recién creadas para el fomento del turismo. Además, que en su desarrollo también participaron empresarios y sus habitantes (Álcala, 1994 y Cárdenas 2014).
} 
expropiaciones "hasta ventas fraudulentas, apropiaciones ilegales o despojos por parte de políticos, consorcios empresarias o caciques aliados a las estructuras de gobierno" (Marín, 2015, p. 16).

Otra de las peculiaridades de las ciudades turísticas de sol y playa es el rápido crecimiento económico, urbano y demográfico que alcanzan en pocos años (Anton, 1992 y Mullins, 1989), lo cual se podrá constatar en el presente documento. Sin embargo, ello se vuelve un problema porque desafortunadamente en México "no existen los suficientes trabajos de casos en donde se examine la sustentabilidad de los productos turísticos y se reconozcan los errores y éxitos del pasado, sobre todo cuando se advierte en los propios destinos la existencia de numerosos problemas" (Gómez, 2003, p. 104). Tema que saldrá a relucir a lo largo del texto.

En este contexto vale la pena preguntarse ¿cómo ha sido el proceso de planeación urbana en México? Debido que ésta es una actividad importante, pues asimismo se debe estipular ¿quién o quiénes son los encargados de ordenar el territorio? Ello con el objetivo de lograr ciudades más competitivas en términos económicos.

Se debe enfatizar que en teoría queda muy bien establecido que el municipio es el encargado de ordenar su territorio. Sin embargo, México al ser un país federalista le corresponde a distintas secretarías de Estado opinar sobre los asuntos locales, dándose una yuxtaposición de tareas. Para ejemplificar, a nivel nacional existen dos programas que se implementan de forma separada y son: el Ordenamiento Ecológico coordinado por la Secretaría de Medio Ambiente y Recursos Naturales (SEMARNAT) y el Ordenamiento Territorial bajo la responsabilidad de la Secretaría de Desarrollo Social (SEDESOL) ${ }^{2}$ (Wong, 2009, p. 3). Así pues, la planeación territorial es tema de ambos programas, aunque el primero lo aborda de manera general y el segundo solamente se limita a las áreas ecológicas.

Así pues, en el tema del ordenamiento territorial en México el reto es mayor porque se deben integrar tanto las cuestiones medioambientales como las sociales en un contexto neoliberal (García y Córdoba, 2008, p. 139). Además, de que estas dos instancias deben entrar en colaboración con órdenes de gobierno estatal y municipal. La tarea se complica aún más cuando los encargados de su funcionamiento no tienen las mismas herramientas técnicas para entablar un diálogo. Ello a pesar de que existe el Comité de Planeación para el Desarrollo del Estado (COPLADE) cuya función es coordinar las relaciones entre los gobiernos federal, estatal y municipal, derivadas de las acciones de planeación. Así como los Comités de Planeación para el Desarrollo Municipal (COPLADEMUN), el cual es un órgano auxiliar del ayuntamiento en temas de planeación. El encargado de integrar al comité es el presidente municipal, por lo que otra de sus debilidades es que existe una fuerte burocratización, poca participación ciudadana y generalmente el tema que se analiza es la implementación de los Fondos que recibe de la Federación, como es el ramo 33.

Además de lo anterior, algunos autores han recalcado que las debilidades de la planeación urbana en México son básicamente cuatro:

\footnotetext{
${ }^{2}$ Uno de los cambios en la actual administración de Enrique Peña Nieto es que se constituyó la Secretaria de Desarrollo Agrario, Territorial y Urbano (SEDATU), otorgándole funciones que anteriormente estaban a cargo de la Secretaría de Desarrollo Social.
} 
1) La ausencia "de participación de la sociedad en la formulación, evaluación, aprobación y seguimiento de los instrumentos de ordenamiento, a pesar de estar estipulada en todos los ejercicios de la consulta pública" (Baños y Cárdenas, 2014, p. 39). Idea que también comparte Graizbord (1999) y Verduzco (2013). Aunque este último autor enfatiza respecto al papel que juegan los actores clave en la toma de decisión.

2) Las deficiencias existentes en la metodología de la planeación urbana, debido a que se basa exclusivamente en la zonificación espacial como instrumento de aplicación de las políticas de ordenamiento territorial (Baños y Cárdenas, 2014, p. 38).

3) La falta de planeación urbana a largo plazo (Vásquez, 2010). Así como el "escaso seguimiento y evaluación a los resultados de actividades y acciones derivadas de la ejecución de los instrumentos de planeación" (Calderón y Orozco, 2009, p. 21).

4) Finalmente y no menos importante es el problema de la corrupción que se gesta y desarrolla entre empresarios y funcionarios públicos en la construcción de proyectos sin respetar la normativa urbana y ambiental (Baños y Cárdenas, 2014; Gómezjara, 1974; Marín, 2015; Ramírez, 1986). Ello genera graves problemas a la ciudadanía y hasta el mismo gobierno de administraciones futuras, al delegar constantemente responsabilidades, así pues, las autoridades replican la consigna "el de atrás paga" (Cabrales, 2010, p. 76).

Enunciado lo anterior, toca el turno de explicar el crecimiento urbano que han registrado tres ciudades mexicanas, tomando en consideración que una de las actividades económicas que ha tenido un mayor auge en los últimos años es el turismo (Clancy, 2001; Everitt, et al. 2008; Pie y Rosa, 2013 y WTO, 2015). Éste "es un potente inductor de cambios en la estructura económica, que se asocian con los desplazamientos masivos de población en algunas temporadas del año" (Hiernaux y Rodríguez, 1991, p. 14). Uno de sus impactos está relacionado con el crecimiento de ciudades existentes o la creación de nuevas (Anton, 1992; Arroyo, 1980; Hiernaux y Rodríguez, 1991; Mullins, 1989).

En otras palabras y como lo comenta Díaz:

El turismo es la actividad que probablemente más cambios ha determinado en el litoral en los últimos años: no solo por la ocupación del mismo y el valor de posición que con él adquiere la primera línea de mar, sino también porque ha dado lugar a infraestructuras y formas urbanas propias: desde los puertos deportivos o las marinas hasta los condominios o los resorts turísticos cerrados, microciudades capaces de ofrecer todos los servicios que se puedan necesitar durante una estancia corta, sin necesidad de salir de ellos. Por otra parte, la estacionalidad propia de la actividad turística que provoca una enorme distorsión entre la dimensión de la ciudad realmente construida y la de la población que vive en ella, lo que afecta a la prestación de servicios y al mantenimiento de las infraestructuras. (2013, p. 195).

\section{CRECIMIENTO URBANO Y PLANEACIÓN URBANA EN CIUDADES TURÍSTICAS DE SOL Y PLAYA EN MÉXICO}

El siguiente apartado dará cuenta de cómo ha sido el proceso de crecimiento urbano y de planeación en las ciudades turísticas de sol y playa seleccionadas. Pero antes es conveniente hacer una breve presentación de las mismas. 


\subsection{Acapulco: contexto histórico y crecimiento urbano}

El municipio de Acapulco de Juárez se ubica en la franja costera del Océano Pacífico, su cabecera municipal cuenta con una superficie de 13658.77 hectáreas, (INEGI, 2010). Su historia se remonta varios siglos atrás, pues durante la Colonia tuvo un papel importante al desempeñarse como puerto marítimo, mediante el que se establecieron lazos comerciales entre Asia y las ciudades de la Nueva España (Oteiza, 1973). Después de la Independencia de México respecto a España se ha señalado que Acapulco se convirtió en un pueblo aislado (Ramírez, 1986). Sin embargo, hoy día se enfatiza que en el siglo XIX éste "tuvo una participación destacada en el sistema de navegación internacional y constituyó una puerta de entrada y salida para los pueblos sureños del estado de Guerrero" (Busto, 2015, p. 287).

Pero la historia de Acapulco volvió a tomar nuevos bríos cuando en 1927 se construyó la carretera Acapulco-Taxco así como la entrada en operación, en 1928, de un aeropuerto (Ramírez, 1986, p. 482). Con dichas obras se estableció accesibilidad a turistas. Asimismo, se sentaron las bases para la llegada de inversiones extranjeras en la construcción de hoteles (Santamaría, 2002, p. 57).

Otros hechos significativos que coadyuvaron al despegue de Acapulco posterior a 1950 fueron: 1) Estados Unidos de América lo vio un espacio propicio para la recuperación de sus excombatientes de la Segunda Guerra Mundial; 2) el financiamiento que hizo el gobierno federal en la construcción de infraestructura y servicios (Ramírez, 1986, p. 483); y 3) la llegada al poder de Fidel Castro en Cuba; lo que obligó a los estadounidenses a buscar otros lugares de esparcimiento (Hiernaux, 2010, p. 104).

Sin embargo, un personaje clave en el origen y desarrollo de Acapulco como destino turístico fue la intervención del entonces presidente de México Miguel Alemán Valdés (19461952) quien dispuso del establecimiento de la Junta Federal de Mejoras Materiales, la cual llevó a cabo obras, tales como:

La pavimentación del centro de la ciudad, con el alineamiento de las calles, captación de aguas, la Calzada Costera, Gran Vía Tropical, el Aeropuerto de Pie de la Cuesta, la Carretera Escénica, la de la Garita a la Fuente de Diana, el nuevo Palacio Federal, el embellecimiento de las playas de Caleta y Caletilla, la Planta Termoeléctrica de Vista Alegre, el Aeropuerto Internacional del Plan de los Amates, entre otras... (Oteiza, 1973, pp. 391 y 392).

Con la llegada de la actividad turística a Acapulco, lejos quedó atrás la imagen de una villa aislada, pues ya para la década de los años sesenta era el principal destino turístico de sol y playa que contaba el país. Su evolución se puede apreciar con los siguientes datos: en 1954 Acapulco recibió 92.694 turistas. En 1960 se registraron 540.100 y para 1972 sumaron 1.5 millones (Ramírez, 1986, p. 491).

Ahora bien, con la llegada de cientos, miles y millones de turistas se requirió de abundante mano de obra para su servicio. Esto generó la atracción de cientos y miles de migrantes que andan en busca de mejores condiciones de vida. Por ello Acapulco ha registrado en los últimos 60 años un alto crecimiento demográfico: En 1900 Acapulco estaba habitado por 4.932 personas, para 1950 eran 28.512 moradores. Para el año 2010 eran 673.479 sus habitantes. Como se ilustra en el siguiente gráfico: 
Gráfico 1. Población de Acapulco de Juárez, Guerrero, 1950-2010

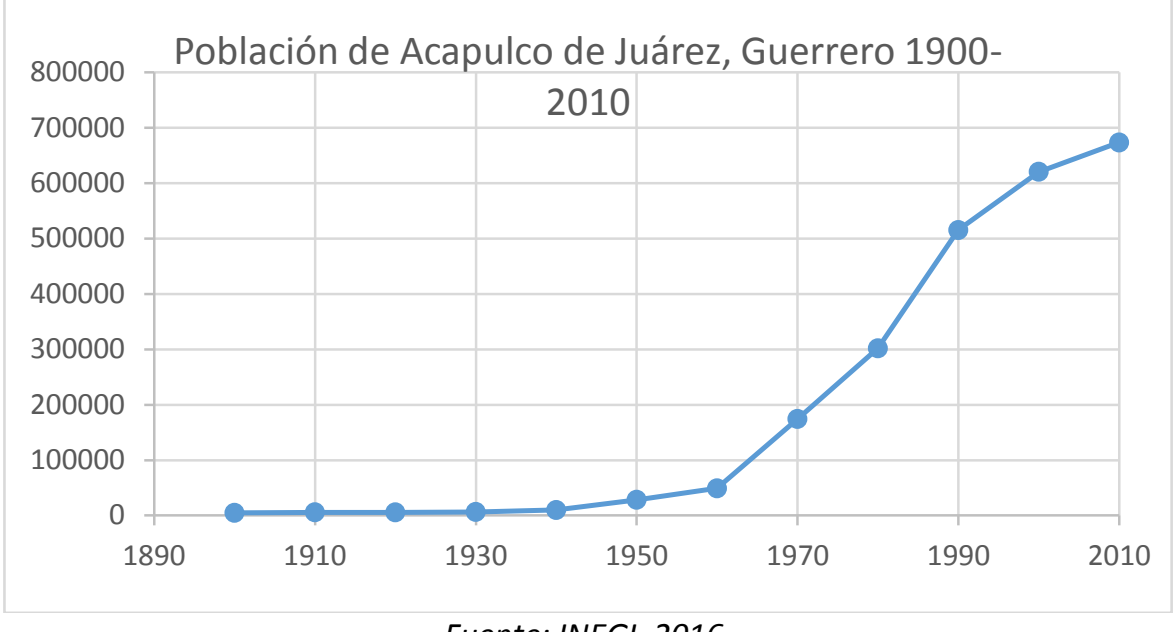

Fuente: INEGI, 2016.

Respecto a la migración en Acapulco, Ricardo Garibay comenta que en la década de los años setenta "llegó gente pobre de Jalisco, Michoacán, Oaxaca, etcétera, donde armaron un jacal con varas y tablones (...) y es así como se crearon zonas perdidas ${ }^{3}$ en cerros y hondonadas, sin luz, sin agua, sin pavimento, sin drenaje" (1979, p. 140). Situación que expondrá la responsabilidad del gobierno tanto estatal como municipal al haber permitido esos asentamientos cuando el lugar fue azotado por el huracán Paulina.

Sin duda alguna existe relación entre el crecimiento demográfico y la expansión de la mancha urbana. Aunque en Acapulco entran en juego más factores, como son: el incremento de hoteles y fraccionamientos así como el surgimiento de colonias populares (Ramírez, 1986, p. 493). Idea que también concuerda Hiernaux, quien además agrega que el modelo residencial en Acapulco. "(...) siempre estuvo presente a todo lo largo de la historia del desarrollo y no particularmente en cantidades menores, sino que más bien resultó subvaluado por las estadísticas locales" (2010, p. 110).

Desafortunadamente en este proceso "el gobierno no asumió el papel de regulador y ordenador de la incorporación de la tierra a usos urbanos en función del futuro de la actividad turística del puerto" (Ramírez, 1986, p. 487). Al contrario, el mismo gobierno expropió y vendió propiedades ejidales ${ }^{4}$ a muy bajos precios. De igual manera, con el paso de los tiempos varios exfuncionarios públicos participaron como compradores, ejemplo de ello fue Miguel Alemán Valdés, quien como inversionista adquirió varios terrenos (Gomezjara citado por Bringas, 1999, p. 17). ${ }^{5}$

En esta tesitura, otro de los personajes clave en la urbanización de Acapulco es la familia Azcárraga (principales accionistas de la empresa de telecomunicaciones Grupo

\footnotetext{
${ }^{3}$ Por zonas perdidas entiéndase un asentamiento irregular.

${ }^{4}$ El ejido es una forma de la tenencia de la tierra en México y es un fruto de la Revolución Mexicana donde a partir de 1915 se hicieron donaciones de tierra de manera particular o colectiva. Una cuestión importante es que en un principio los ejidatarios solamente tenían derecho a utilizar la tierra y no podían venderla, situación que cambió con la reforma al artículo 27 de la Constitución Política de los Estados Unidos Mexicanos en el año de 1992.

${ }^{5}$ Respecto a la expropiación del uso del suelo que se ha hecho en Acapulco, Eurosia Carrascal y Graciela Pérez comentan varios aspectos importantes: 1) que una compañía estadounidense antes de 1940 se apropió de los terrenos que ocupaban los parajes de La Langosta, Manzanillo, Playa Larga, El Ahorcado y Tambuco, hasta las playas de Caleta y Caletilla y 2) que en esas mismas fechas se expropiaron 740 ha del ejido de Icacos, de las cuales se destinaron 665 para la instalación del fraccionamiento Costa Azul (1998, p. 113).
} 
Televisa) "que era dueña de prácticamente todos los terrenos desde la glorieta de la Diana hasta la base naval de Icacos" (Fernández y Paxmon citado por Valenzuela y Coll, 2010, p. 172).

Ahora bien, son varios los autores que dividen la historia de Acapulco en tres momentos, los cuales se plasman en el territorio: tradicional, dorado y diamante. El primero tuvo su periodo de auge entre 1930 y 1960. En éste se concentran tanto las actividades turísticas como las actividades comerciales, administrativas y residenciales. A él acuden las personas de bajos recursos. Las principales problemáticas de dicho lugar son la escasa clientela, en las playas existen vendedores ambulantes y basura. En otras palabras, es una zona deprimida (Valenzuela y Coll, 2010, p. 184). El segundo, el Acapulco dorado se encuentra en el centro de la bahía, cuenta con la mejor infraestructura y el mejor equipamiento de servicios turísticos y urbanos (Valenzuela y Coll, 2010, p. 184).

El tercero, la zona diamante, donde coexisten diferentes usos del suelo, tales como: turístico de bajo impacto, residencial, rural-urbano, industrial, de cultivos, huertos y área protegida. Por varias razones hoy día se constituye como un centro de lujo especializado (Valenzuela y Coll, 2010, p. 186).

Ahora bien, en esta división respecto a la historia de Acapulco, Hiernaux considera que es un gran mito, pues el paso de tradicional a dorado obedeció únicamente para promover las nuevas instalaciones a lo largo de la bahía y desacreditar lo que ya se había construido (2010, pp. 105 y 106).

En el siguiente mapa se puede ilustrar el crecimiento urbano que ha registrado Acapulco en el periodo que abarca de 1930 al 2014. Los datos son los siguientes:

Cuadro 1. Crecimiento urbano de Acapulco, Guerrero, 1930-2014

\begin{tabular}{|c|c|}
\hline Año & Hectáreas urbanizadas (acumuladas) \\
\hline 1930 & 52.501 \\
\hline 1981 & 1775.630 \\
\hline 1998 & 5387.290 \\
\hline 2014 & 16574.621 \\
\hline
\end{tabular}

Fuente: Cálculos propios a partir de los polígonos urbanos de INEGI con base en las cartas mencionadas en el mapa y las herramientas de ArcMap.

En 84 años se han urbanizado un total de 16522 hectáreas. Llama la atención que el último periodo de estudio, que comprende de 1998 a 2014, se urbanizaron 11 mil hectáreas, que representan el $67 \%$ del total. Ello demuestra que Acapulco recibe cuantiosas inversiones. Si bien es cierto que el destino ya no es visitado en su mayoría por turistas extranjeros, tampoco se debe negar el hecho de que cada fin de semana se da cita el turismo doméstico en el lugar, sobre todo, la gente que vive en la zona metropolitana del Valle de México. De igual manera, se deben señalar que participan en el mercado inmobiliario los estadounidenses y canadienses, quienes muestran interés en comprar una segunda residencia en Acapulco (Hiernaux, 2010 y Valenzuela y Coll, 2010). 
Mapa 1. Crecimiento urbano de Acapulco, Guerrero, 1930-2014

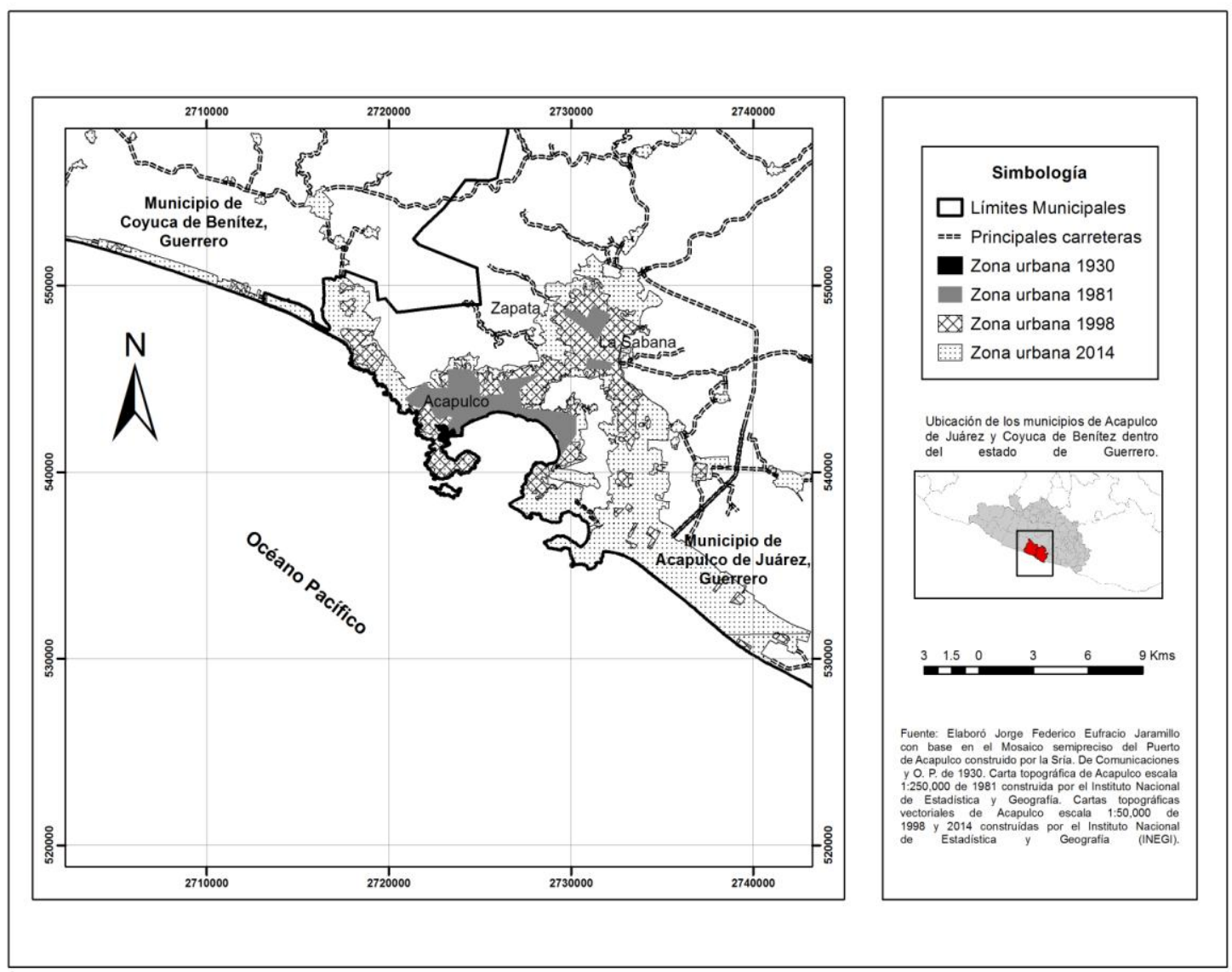

En el mapa se ilustra que Acapulco no cuenta con suelo para urbanizar en su franja costera, por dicha razón se ha dado paso a una urbanización de tipo nodal y la cual se extiende a los municipios contiguos como Coyuca de Benítez y San Marcos. Ello ha traído consigo la conformación de una zona metropolitana que cuenta con varios centros, como lo han señalado varios organismos institucionales como SEDESOL, el Consejo Nacional de Población, (CONAPO) y el Instituto Nacional de Estadística y Geografía (INEGI) (2004).

\subsubsection{Planeación urbana en Acapulco}

En términos generales se puede señalar que la planeación urbana en Acapulco ha sido indicativa con algunos intentos tardíos de corregir algunas anomalías. En su historia se han dado algunos intentos valiosos por ordenar su territorio. Uno de ellos fue el que se realizó con el gobierno de Pascual Ortiz Rubio en la década de los años de 1930 (Valenzuela y Coll, 2010, p. 171). Otro de éstos acaeció en 1976 cuando se creó el Fideicomiso Acapulco (FIDACA) y el cual se enfocó en la construcción de varias obras de urbanización (Ramírez, 1986, pp. 505-506). Pero con dichas acciones Acapulco se dividió en dos: "el primero ocuparía la bahía y estaría dedicado exclusivamente al turismo y el segundo sería la ciudad de los pobres y de las funciones urbanas necesarias pero que es conveniente ocultar a los ojos de los visitantes, sobre todo extranjeros" (Ramírez, 1986, p. 506). 
Es importante recalcar que desde la década de 1980, la ciudad muestra signos de agotamiento por la terrible contaminación que sufren sus ecosistemas (Ramírez, 1986, Carrascal y Pérez, 1998 y Bringas 1999). Así como por la violencia que ha azotado en los últimos años a la región. De enero a marzo de 2016 en Acapulco se habían ejecutado a 204 personas, además de presentarse varios enfrentamientos entre integrantes del crimen organizado y la policía federal en la avenida Miguel Alemán (Aguilar Excélsior, 2016).

Otra cuestión que se debe mencionar es que las ciudades turísticas de sol y playa son altamente vulnerables a los fenómenos meteorológicos y Acapulco no es la excepción. Así pues, con la llegada del huracán Paulina en 1997, se despertó el interés por la planeación urbana, debido a que murieron 207 personas, 200 desaparecieron y 52.000 perdieron su vivienda (Toscana, 2003, p. 65). Ello porque "los lechos de inundación de los ríos estaban poblados, a causa de la explosión demográfica en Acapulco y a la escasa planeación urbana" (Toscana, 2003, p. 67). Problemática que se agudizará a un más en los próximos años, pues hoy uno de los retos que enfrentan las ciudades turísticas ubicadas en los litorales son: "la elevación del nivel del mar y el aumento de frecuencia e intensidad de las tormentas que amenazan destinos e infraestructura" (Ramírez et al., 2015, p. 129).

Ante dicha situación el gobierno federal, a través de varias instituciones, elaboró en el año de 2001 un nuevo Plan Director Urbano pero tuvieron que pasar cuatro años más para su promulgación. Aunque, después no se podía aplicar porque carecía de Reglamento (Ramírez, 2009, pp. 1 y 54). Así pues, lamentablemente los problemas urbanos de Acapulco no recaen en la falta de planeación urbana ${ }^{6}$ sino en la corrupción que se gesta entre autoridades y empresarios (Ramírez, 2009). En este sentido, Marín señala que el gobierno del estado durante el mandato de Francisco Ruiz Massieu (1989-1993) facultó a través del organismo Promotora Turística a expropiar terrenos ejidales, en Puerto Marqués, La Poza, Copacabana, Plan de los Amates, Alfredo V. Bonfil y Lomas de Chapultepec, para posteriormente entregarlos en venta a inversionistas (2015, p. 19).

\subsection{Cancún: localización geográfica y crecimiento urbano}

Cancún se localiza en la franja costera frente al Mar Caribe. Tiene un extensión territorial de 13.027,02 hectáreas (INEGI, 2010). Su proceso de gestación como ciudad responde a las acciones emprendidas por el gobierno federal por incentivar el desarrollo en algunos territorios del litoral mexicano en la década de los años sesenta, como ya se indicó. Para su construcción ocupó una superficie de 12.700 ha, de las cuales el 55\% son tierra firme y el resto, terrenos inundables (Aldape, 2013, p. 228). En otras palabras, "Cancún es la primera ciudad del país que nace totalmente planificada. Desde el punto de vista urbano se rompe con el patrón de la plaza central" (Castillo, 2011, p. 26).

Específicamente el proyecto de Cancún se gesta para cumplir cuatro objetivos. Uno de ellos es:

\footnotetext{
${ }^{6}$ Algunos de los planes que han tratado de regular el territorio de Acapulco son: Primer Plano Regulador de la Ciudad, 1889; Actualización del Plano Regulador, 1945; se creó la Comisión de Planificación Regional de Acapulco, 1951; el Plan de Acapulco, 1970; la constitución del Fideicomiso Acapulco, FIDACA, 1980; el Plan Director Urbano de Acapulco, 1980; el Reglamento de Zonificación y Usos del Suelo del Plano Regulador del Municipio de Acapulco, 1985; el Plan Director Municipal 1988; el Plan Parcial de Acapulco Diamante, 1990; el Plan Parcial de Desarrollo Urbano; la Declaratoria de Usos y Destinos del Suelo para la Zona del Acapulco Tradicional, 1991; el Plan Parcial de Desarrollo Urbano de Acapulco Dorado, 1991; el Plan Director Urbano, 1993; y el Plan Director Urbano de la Zona Metropolitana de Acapulco, 2001 (Ramírez, 2009, pp. 97-98).
} 
... la creación de nuevas fuentes de trabajo en zonas potencialmente turísticas y en las que existen núcleos importantes de población rural o semi rural de bajos ingresos. Estas zonas presentan por lo general escasas posibilidades para el desarrollo de otras actividades productivas. (Fonatur, citado por García, 1979, p. 81).?

Dicho objetivo hasta cierto punto se ha cumplido, pues Cancún es uno de los lugares que más crecimiento demográfico ha registrado en el país debido a que ofrece empleo de manera permanente, aunque hace falta, por ejemplo, elaborar un estudio más minucioso respecto a la calidad de las plazas laborales que se ofrecen. En el siguiente gráfico se puede ilustrar el crecimiento demográfico que ha registrado en los últimos 40 años el municipio de Benito Juárez, en Quintana Roo y del cual Cancún forma parte. Para 1970 tenía una población de 12 mil habitantes y para el 2010 eran 661 mil sus moradores.

\section{Gráfico 2. Población de Benito Juárez, Quintana Roo, 1980-2010}

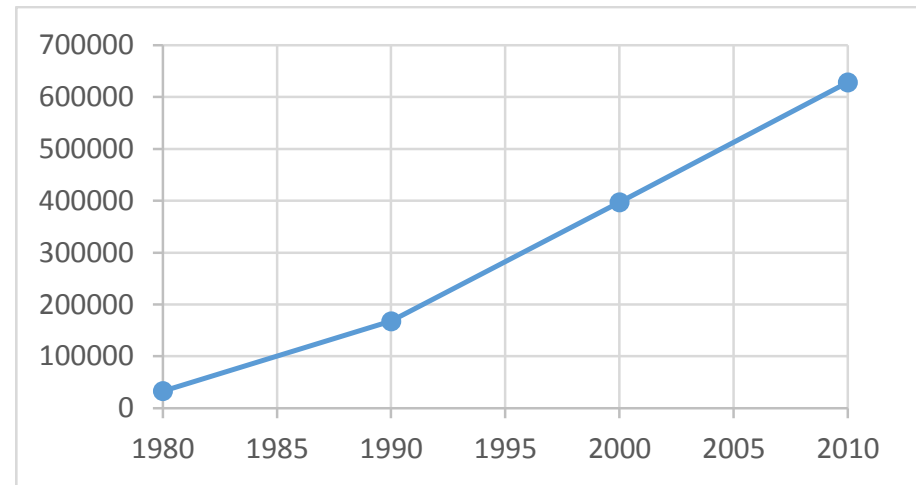

Fuente: INEGI, 2016.

Ahora bien, es importante advertir que desde un principio en el plan maestro de Cancún se crean "áreas sociales diferenciadas y especialmente separadas" (Arnaiz y César, 1994, p. 61). Ésta son: 1) la parte de la franja costera donde se ubicarían los hoteles, 2) el alojamiento para los administradores y 3 ) la vivienda de los trabajadores, en general.

Aunque la parte destinada a los trabajadores, conocida como Regiones, también ha crecido en proporciones desmedidas, por dicha razón se observa la carencia de servicios públicos, educativos y de salud (Castellanos y París, 2005, p. 139).

En este contexto, Cancún es un claro ejemplo de la segregación socio-espacial que se vive en las ciudades turísticas de sol y playa (Castellanos y Pombo 2005; Castillo 2011; García, 1979, Marín 2008; Oehmichen 2010 y 2013; Torres y Momsen, 2005). Pero lo lamentable de la situación es que a pesar de que fue planeada desde un principio no evitó que se agudizaran las condiciones de segregación propias de las ciudades sino que las ha

\footnotetext{
${ }^{7}$ Los otros tres objetivos son: el impulso al desarrollo regional mediante el estímulo a nuevas actividades agrícolas, industriales y artesanales en la zona; la mejoría y diversificación de los centros de atractivo turístico del país para mantener e incrementar la corriente turística del exterior de México; y el incremento a corto y mediano plazo de los ingresos de divisas que cuenta corriente de la balanza de pagos por este concepto, estimando que este renglón es más susceptible de aumento a corto plazo que otros rubros, tales como las exportaciones de productos de todas clases (Fonatur, citado por García, 1979, pp. 80 y 81).
} 
agravado al reforzar la separación entre las distintas zonas y estimular los modos de consumo de cada una de ellas (Castillo y Villar, 2011, pp. 95 y 96).

Así pues, la consolidación turística de Cancún rebasó las fronteras del polígono original, es por ello que para el año de 1990 fue necesaria la construcción del Corredor Cancún-Tulum (mejor conocido como la Riviera Maya), que pronto igualó en crecimiento al polo de desarrollo inicial (Marín, 2008, p. 122).

Otros de los problemas que enfrenta Cancún son: 1) la contaminación que sufren algunos de los ecosistemas; 2) la falta de accesos a la playa (Castillo, 2011, p. 26); y 3) el conflicto entre ejidatarios y el gobierno para detener la venta ilegal de terrenos (Torres y Momsen, 2005, p. 330). Por lo anterior se considera a Cancún como un polo de subdesarrollo.

Algunos aspectos positivos de Cancún son: 1) representa la principal fuente de ingresos de turismo planificado de México (Aldape, 2013, p. 227). 2) Cuenta con 26.4 miles de cuartos de hotel, y otro tanto ( 24.3 miles) se han construido en la Riviera Maya, cuando únicamente se había pensado edificar 6000 habitaciones en Cancún (Oehmichen, 2010, p. 47). Se han registrado 3.1 millones de huéspedes, su aeropuerto es el segundo más importante a nivel nacional, por la movilización de pasajeros (Sectur, citado por Jiménez y Sosa, 2007). De igual manera, se debe destacar el hecho de que el índice de Desarrollo Humano del Programa de las Naciones Unidas para el Desarrollo (PNUD) califica al municipio de Benito Juárez como "Muy alto"; asimismo, esta nota se reafirma en los indicadores de Marginación del Consejo Nacional de Población (CONAPO).

En el siguiente mapa se puede ilustrar el crecimiento urbano que ha registrado Cancún en los últimos 22 años. El punto de partida es el año de 1975 con 277 hectáreas urbanizadas que señaló Castillo (2011, p. 92). En 32 años se han urbanizado 14 mil hectáreas. Llama la atención el último periodo de estudio, que comprende del año 2008 al 2014 pues se urbanizaron 7 mil hectáreas (Cálculos realizados a partir de los polígonos urbanos de INEGI con base en las cartas mencionadas en el mapa y las herramientas de ArcMap). Este crecimiento urbano ha dado paso de una ciudad lineal a otra de tipo nodal y donde también involucra a municipios vecinos.

Cuadro 2. Crecimiento urbano de Cancún, Quintana Roo 1982-2014

\begin{tabular}{|c|c|}
\hline Año & Hectáreas urbanizadas (acumuladas) \\
\hline 1982 & 675.73 \\
\hline 2000 & 3.351 .51 \\
\hline 2008 & 7.368 .23 \\
\hline 2014 & 14.495 .09 \\
\hline
\end{tabular}

Fuente: Cálculos realizados a partir de los polígonos urbanos de INEGI con base en las cartas mencionadas en el mapa y las herramientas de ArcMap. 
Mapa 2. Crecimiento urbano de Cancún, Quintana Roo, 1982-2014

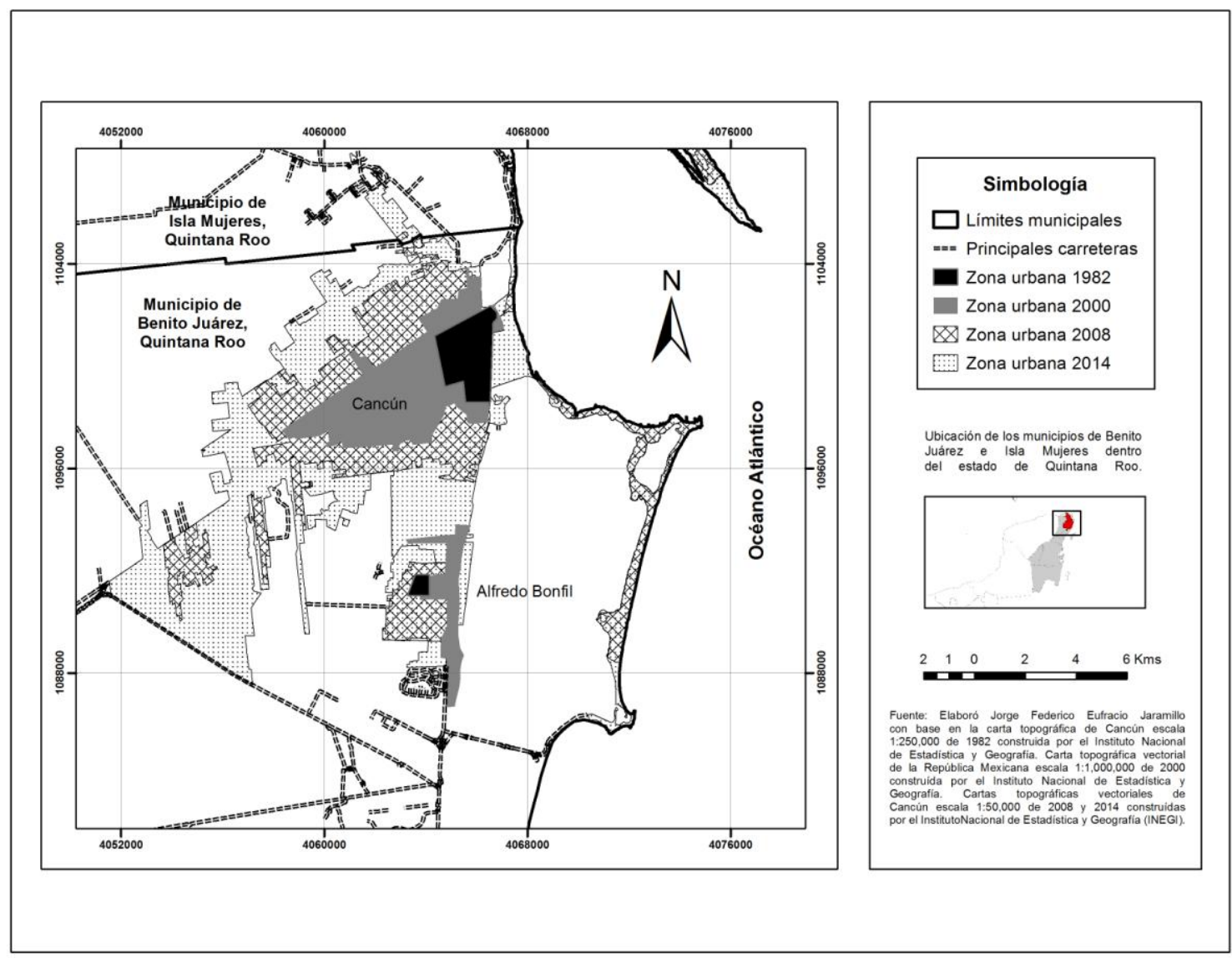

\subsubsection{Planeación urbana en Cancún}

Infratur y Banco de México dieron a conocer el Plan Maestro de Cancún el 10 de agosto de 1971. Los demás planes y como lo comenta Carlos Veloz han sido los siguientes:

En 1985 se publicó el primer Plan Director de Desarrollo Urbano, y el cual se actualizó en 1993. En 1995 el documento se renovó con la participación de los gobiernos estatal y municipal. Para 1998 se publicó el primer Programa Parcial para incorporar la Reserva Sur al desarrollo urbano. En el año 2000 se publicó el Programa Parcial de la Reserva Norte, en el año 2001 se zonificó Puerto Cancún. Mientras que para el año 2003 se publicó el Programa Parcial de la Tercera Etapa de la Zona Hotelera y en el 2004 se publica por primera vez el Programa Parcial del Polígono 11. En el 2005 surgió el Plan de Desarrollo Urbano. En el 2006 se publicó el Programa de Ordenamiento Ecológico Local (POEL). Actualmente, Cancún cuenta ocho Programas Parciales vigentes y anexos al Programa de Desarrollo Urbano (manuscrito).

Uno de los aspectos positivos de Cancún es que cuenta desde el año de 2001 con el Instituto de Planeación de Desarrollo Urbano (IMPLAN). Éste es un Organismo Público Descentralizado especializado en la Planeación de Desarrollo Urbano del municipio y el cual no está sujeto a los cambios electorales de las administraciones gubernamentales. Las 
funciones son: a) la actualización y realización de los distintos planes y programas de desarrollo urbano y medio ambiente (Calderón y Orozco, 2009, p. 22).

En resumen, Cancún en su corta vida ha contado con varios instrumentos de planeación pero como lo comentan Arnaiz y César, la problemática del lugar recae en la crisis de gestión y en la irresponsabilidad de los empresarios que dejan el problema al Estado y no tanto por la falta de planeación (1994, p. 63). A pesar de que existe el plan maestro definido por Fonatur y se cuenta con diversos instrumentos de planeación, los ayuntamientos cuentan con la facultad de modificar los usos del suelo establecidos para que se construyan inmuebles fuera del marco legal. Además, los citados autores destacan que "no se han respetado los planes originales de manejo y destino del suelo, pues una vez que creció la actividad turística en el lugar se vendieron los espacios para áreas verdes e incluso se edificó sobre humedales contaminando de manera irreversible la laguna Bojórquez" (1994, p. 61).

\subsection{Puerto Vallarta contexto histórico y crecimiento urbano}

Puerto Vallarta se localiza frente al Océano Pacífico, cuenta con 4695.38 hectáreas (INEGI, 2010). Se fundó el 12 de diciembre de 1851 cuando un grupo de pescadores se instalaron a orillas del río Cuale y denominaron al poblado Las Peñas de Santa María de Guadalupe (Munguía, 1997, p. 100). La tranquilidad y la quietud van durar hasta la década de los años sesenta del siglo XX que es -cuando se impulsó el turismo como actividad económica-, por iniciativa de los tres órdenes de gobierno, empresarios y pobladores. A partir de entonces Puerto Vallarta va a registrar un importante dinamismo económico, el cual se reflejará en el alto crecimiento demográfico que posee, como se puede observar en el siguiente gráfico. En 1960 contaba con 15 mil moradores y para el año 2010 eran 255 mil sus habitantes.

Gráfico 3. Población de Puerto Vallarta, Jalisco, 1900-2010

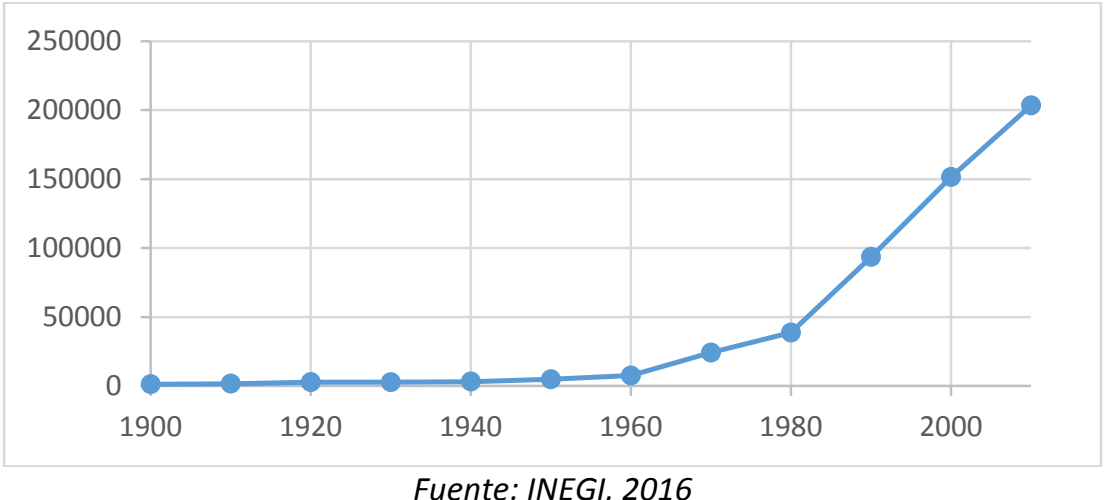

Como el crecimiento demográfico se plasma en la mancha urbana, las cifras que así lo constatan son: en el año de 1977 Puerto Vallarta contaba con 372.31 hectáreas urbanizadas, en 1996 eran 2499.99 y para el 2010 sumaron 7 325.21. Llama la atención que en catorce años casi se triplican las hectáreas urbanizadas. 
Cuadro 3. Crecimiento urbano de Puerto Vallarta, Jalisco 1977-2014

\begin{tabular}{|c|c|}
\hline Año & Hectáreas urbanizadas (acumuladas) \\
\hline 1977 & 372.31 \\
\hline 1996 & 2.499 .99 \\
\hline 2010 & 7.325 .21 \\
\hline
\end{tabular}

Fuente: Cálculos realizados a partir de los polígonos urbanos de INEGI con base en las cartas mencionadas en el mapa y las herramientas de ArcMap.

En el siguiente mapa se puede ilustrar el crecimiento urbano que ha registrado Puerto Vallarta en los últimos 33 años. Para el 2010 este destino ya no contaba con hectáreas para urbanizar en su franja costera. Por dicha razón su dinamismo económico ha alcanzado al territorio del estado de Nayarit, específicamente al joven municipio de Bahía de Banderas. Así como en su interior, dando paso a una ciudad de tipo lineal a la nodal. Es por ello que SEDESOL, CONAPO e INEGI la delimitaron como una zona metropolitana (2004).

Mapa 3. Crecimiento urbano de Puerto Vallarta, 1977-2010

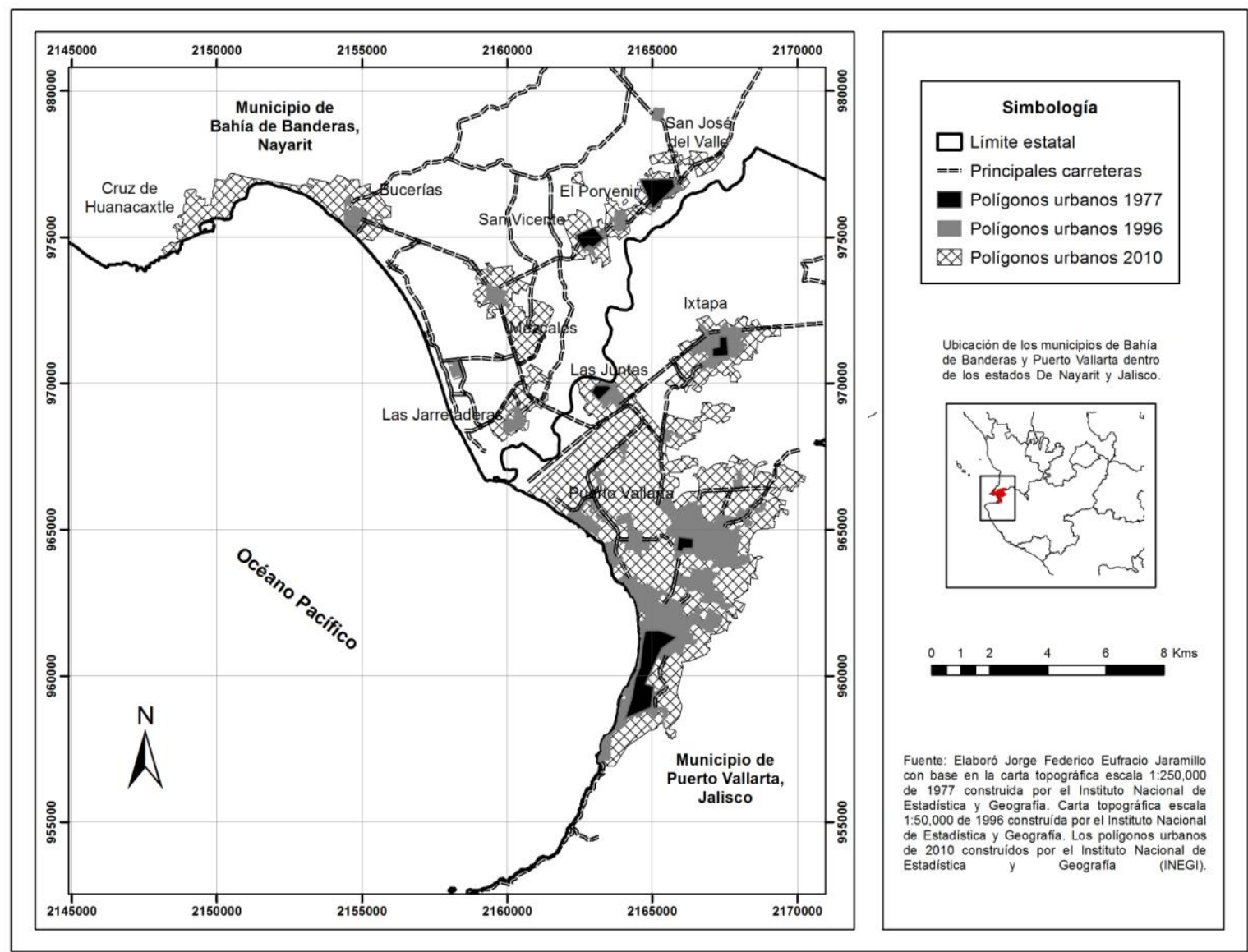

\subsubsection{Planeación urbana en Puerto Vallarta}

En el año de 1975 la Junta General de Planeación y Urbanización del estado de Jalisco elaboró el Plan General Urbano de Puerto Vallarta. Su actualización ocurrió en 1982 y fue con el propósito de adecuar el documento a los cambios experimentados en la ciudad 
(Baños, 2010, pp. 90 y 91). Otra actualización sucedió en 1989 y el cual incorporó algunos conceptos interesantes, tales como: Clasificación de Áreas, Usos y Destinos y las modalidades de Utilización del Suelo (Baños, 2010, p. 92).

En Puerto Vallarta en el año de 1997 se aprobó el Plan de Desarrollo Urbano en donde se establecieron las normas de control para el aprovechamiento y/o utilización del suelo. Es importante mencionar que dicho plan ha normado el crecimiento urbano de Puerto Vallarta en los últimos 19 años, por lo que se ha vuelto obsoleto pues ya no responde a las necesidades actuales de la sociedad vallartense (Baños, 2010, pp. 94 y 95). Ha habido tres intentos por actualizar el Plan de Desarrollo Urbano (2003, 2006 y 2009). Sin embargo, las iniciativas no han prosperado porque se han enfrentado al rechazo de algunos sectores de la sociedad civil, al grado que ésta ha interpuesto varios juicios administrativos en contra de la autoridad.

La inconformidad ciudadana recae en la pretensión de urbanizar en espacios que no son susceptible para ello (Baños, 2010, p. 102). A pesar de que la sociedad vallartense ha obtenido sentencias favorables en varios juicios, el gobierno ha otorgado licencias de construcción sin respeto a la normatividad, por ejemplo se encuentra el caso del desarrollo del Grand Venettian, "el cual obtuvo un cambio irregular de uso del suelo, que pasó de ser turístico hotelero a habitacional, y que actualmente se dirime administrativamente" (Chavoya, et al., 2010, p. 77). Asimismo, también se encuentra el caso del estero El Salado, del cual en el año 2000 se aprobó su Plan de Manejo de Área Natural Protegida (ANP) y Desarrollo de la Áreas Colindantes y en el cual se estableció una expropiación de 239 hectáreas por causa de utilidad pública, para dar paso a la construcción del centro de convenciones (Gómez, 2003, p. 115).

En este contexto Basilio Verduzco comenta que en Puerto Vallarta "los actores locales han decidido no avanzar en la construcción de acuerdos para guiar el proceso de desarrollo de la ciudad" (2013, p. 22). Ello es de suma importancia lograr y lo es aún más en una ciudad que tiene como vocación económica la actividad turística de sol y playa. Así pues, Puerto Vallarta necesita ser competitiva e innovarse para situarse en el mapa turístico internacional.

Los funcionarios públicos de Puerto Vallarta están conscientes de la situación, pues en el año de 2013 enfatizaron que "los problemas principales a los que se enfrentaban para el ordenamiento territorial y la planeación urbana del municipio estaban vinculados a la incompatibilidad entre los planes y la dinámica real de transformación de la ciudad, sobre todo en lo relativo a los usos del suelo" (Olivares, 2014, pp. 529-530).

No obstante, el problema se agudiza cuando no se tiene un Plan de Desarrollo Urbano actualizado, ello se puede observar cuando por vía infomex ${ }^{8}$ se le preguntó al municipio de Puerto Vallarta: “¿cuántas hectáreas se han urbanizado en los últimos 45 años?", a lo que la Dirección General de Ecología y Ordenamiento Territorial respondió que: "no cuenta con el dato exacto", pero tampoco ofreció una estimación de la urbanización. Al externarle la misma pregunta a la SEDATU, contestó que: "no disponía de esa información" y que la pregunta debía remitirse al gobierno municipal.

\footnotetext{
${ }^{8}$ Sistema virtual dependiente del Instituto Nacional de Transparencia, Acceso a la Información y Protección de Datos Personales (INAI).
} 


\section{DINÁMICAS COMUNES Y DIVERGENTES EN LAS CIUDADES DE ESTUDIO}

Dentro de las dinámicas comunes en las ciudades de Acapulco, Cancún y Puerto Vallarta se debe señalar el alto crecimiento demográfico que han registrado en los últimos años. Sin duda, ello se explica porque estos polos turísticos son captadores de mano de obra de mexicanos que andan en busca de mejores condiciones de vida.

Ahora bien, el crecimiento demográfico se plasmará en el territorio y en esta vorágine entran en juego tanto el ramo hotelero como las empresas inmobiliarias. Así pues, Acapulco a pesar de no ser visitada por extranjeros, registrar un gran número de actos violentos, todavía mantiene un rápido ritmo de urbanización. En el periodo que va de 19982014, se contabilizaron once mil hectáreas urbanizadas, como ya se indicó. En esta dinámica ha estado presente el gobierno al expropiar tierras ejidales y venderlas a empresarios o exfuncionarios públicos a bajos precios, en perjuicio de la gente local (Marín, 2015). El mismo fenómeno ocurre en Puerto Vallarta.

Llama la atención que dicha dinámica también está presente en el caso Cancún, una ciudad planeada donde sus límites únicamente quedaron en un plan, no en la realidad. Con lo anterior se demuestra que hoy día las principales ciudades turísticas de México se siguen urbanizando rápidamente, alcanzando a municipios contiguos dando paso a la conformación de zonas metropolitanas.

A pesar de que el proceso de gestación de las ciudades turísticas estudiadas no es el mismo, pues como ya se indicó Acapulco, es de corte tradicional, Cancún es un CIP que nace de la planeación de un proyecto federal y Puerto Vallarta que se cataloga en una fase intermedia, en los tres asentamientos humanos el crecimiento urbano lo dicta el mercado con la integración de varios actores (formales, como las inmobiliarias; informales, como los paracaidistas, públicos y privados). Lamentablemente en esta dinámica se observan procesos de expropiación de tierras ejidales.

En cuanto a los mecanismos de planeación que se han elaborado en las ciudades estudiadas, era de esperarse que la más calificada en dicho rubro sea la de Cancún, pero no por ello quiere decir que ésta se cumpla. En el caso de Acapulco también se han hecho varios esfuerzos, el último se debió por la urgente necesidad de resguardar a la población ante la entrada de varios fenómenos meteorológicos. La situación en Puerto Vallarta es preocupante pues han pasado 19 años y sus actores no se han puesto de acuerdo para ordenar el territorio. Ello resulta apremiante resolver para lograr desarrollos turísticos sustentables, en esta tesitura concuerdan Núñez y Scarstascini, 2010; Gómez, 2012. Pero desafortunadamente ello no sucede así, pues "la sustentabilidad, al ser un proyecto de crecimiento económico más que de desarrollo humano, tiene al Estado como su principal obstáculo" (Chávez et al., 2013, p. 20).

Otra característica en común de las ciudades seleccionadas es la segregación socioespacial que se vive en ellas. Situación que se recrudece aún más por posición económica, pues son abismales las diferencias en el espacio ocupado y vivido por los visitantes, nativos y migrantes con bajos recursos económicos. A pesar de que han tenido un proceso de nacimiento diferente. 


\section{CONCLUSIÓN}

En el documento se pudo observar que las tres ciudades estudiadas (Acapulco, Cancún y Puerto Vallarta) han registrado un alto crecimiento urbano, debido en gran medida al auge de la actividad turística. Así en pocos años, han pasado de villas aisladas, lugares inhóspitos y pueblos pesqueros a centros turísticos de carácter internacional.

Los datos presentados indican un incremento de las hectáreas urbanizadas en los últimos años, independientemente de que si se trata de una ciudad tradicional como Acapulco, destino turístico que ha pasado por varias etapas de renovación o como Cancún, ciudad que nace expresamente para dar cabida a las actividades turísticas y el cual como destino turístico se encuentra en una fase de esplendor. Por su parte, Puerto Vallarta se ubica en una etapa intermedia, entre la tradicional y el CIP.

Otra característica de estas ciudades es que en pocas décadas han agotado sus hectáreas urbanizables en la franja costera, pasando de un modelo de tipo lineal al nodal. Además, de que el crecimiento urbano se ha extendido a municipios vecinos. De ahí la razón por qué dichos centros urbanos han dado pasó a la conformación de tres nuevas zonas metropolitanas con vocación turística de sol y playa en el país.

A pesar de que México cuenta con una larga tradición en el tema de la planeación urbana, se observa en las ciudades analizadas la ausencia de su aplicación. Pero el problema no es tanto de falta de planes, sino de gestión y de corrupción. En el crecimiento urbano de los territorios turísticos de sol y playa convergen varios procesos y actores: Por un lado se ubican los intereses voraces de los dueños de los hoteles así como de las empresas inmobiliarias y por el otro lado existe la invasión y venta de terrenos ejidales.

La situación en Puerto Vallarta es todavía más complicada, pues no ha actualizado su Plan de Desarrollo Urbano a los requerimientos actuales. Una de las razones es que el gobierno municipal pretende urbanizar en áreas no propias para ello. Ante lo cual la sociedad civil se ha organizado interponiendo varios juicios administrativos. Sin embargo, hace falta que todos los actores de dicha ciudad alcancen acuerdos para el beneficio de la sociedad en general.

Se debe enfatizar que en este contexto de alto crecimiento urbano y demográfico que se presenta en las ciudades turísticas de sol y playa, existe el de la escasez de recursos económicos por parte del gobierno local para dotar de servicios públicos a sus habitantes. Por dicha razón y otras más, como la dirección de las políticas urbanas, las tres ciudades estudiadas, a pesar de que cuentan con diferentes procesos de gestación, la segregación socioespacial que se vive en ellas es una constante.

Por último, es de suma importancia enfatizar que las ciudades turísticas de sol y playa Acapulco, Cancún y Puerto Vallarta alcancen un ordenamiento territorial sustentable, pues de ello depende que permanezcan en el mercado turístico, el cual cada día es más competido. Pero, además, de no hacerlo, corren el riesgo de ser más vulnerables ante los retos que plantea en la actualidad el cambio climático. Para ello es importante la incorporación de ideas y acciones de sus habitantes. 


\section{REFERENCIAS BIBLIOGRÁFICAS}

Aguilar, R. (2016). Violencia imparable en Guerrero, asesinan a 38 personas en Acapulco. México: Excélsior. Recuperado de: http://www.excelsior.com.mx

Álcala, G. (1995). Los pescadores del litoral del Occidente de México y el turismo. Estudios Jaliscienses, Zapopan: El Colegio de Jalisco, 20, 27-44.

Aldape, G. (2013). La configuración del espacio turístico en Cancún, México. En R. Pié i Ninot, C. y J. Rosa Jiménez (Eds.), Turismo líquido (pp. 228-245). Barcelona: Instituto Hábitat Turismo Territorio a través de Iniciativa Digital Politècnica (UPC).

Anton, S. (1998). La urbanización turística. De la conquista del viaje a la reestructuración de la ciudad turística. Documents d'Analisi Geográfica, 32, 17-43.

Arnaiz S., y Dachary A. (1994). "Cancún: los impactos del turismo". Ciudades, 24, 60-64.

Arroyo, F. (1980). Ordenación urbana y especulación turística en Cullera (Valencia). Estudios Geográficos, 161, 383-412.

Baños, A. (2010). Arquitectura y urbanismo en Puerto Vallarta. Una mirada hacia a la construcción turística de litoral. Puerto Vallarta: Astra Ediciones.

Baños, A., y Cárdenas E. (2014). El Federalismo y la planeación urbana en México. Guadalajara: Instituto de Estudios del Federalismo.

Bringas, N. (1999). Políticas de desarrollo turístico en dos zonas costeras del pacífico mexicano. Región y Sociedad, 17, 4-51.

Busto, K. (2015). Acapulco en la segunda mitad del siglo XIX. ¿Estancamiento o desarrollo portuario? En G. Pinzón Ríos y F. Trejo Rivera (coords.), El Mar: percepciones lecturas y contextos. Una mirada cultural a los entornos marítimos (pp. 267-287). México, D.F.: UNAM, Instituto de Investigaciones Históricas, Instituto Nacional de Antropología e Historia.

Cabrales, F. (2010). El de atrás paga: El modelo metropolitano de Guadalajara. En O. Urquidez (coord.), La reinvención de la metrópoli (pp. 75-96). Zapopan: El Colegio de Jalisco.

Calderón, J. y Orozco M. (2009). Planeación y modelo urbano: El caso de Cancún, Quintana Roo. Quivera, Universidad Autónoma del Estado de México, 2(2), 18-34.

Cárdenas, E. (2014). Dinámicas demográficas en tres zonas metropolitanas de México: Acapulco, Cancún y Puerto Vallarta. Espacios Públicos, Universidad Autónoma del Estado de México, 40, 69-93.

Carrascal, E. y Pérez G. (1998). Ocupación territorial y deterioro ambiental ocasionado por la expansión urbano-turística en Acapulco, Guerrero. Investigaciones Geográficas, 37, 111124.

Castillo, O y Villar A. (2011). La conformación del espacio urbano de Cancún: una aproximación al estudio de la segregación socio-espacial, Quivera, 13(1), 83-101.

Castillo, O. (2011). Segregación socioespacial en Cancún: 1990-2010 tres ciudades en una misma. Provincia, 26, 11-31.

Chávez R., Andrade E., Espinoza R. (2013). Turismo y desarrollo sustentable: contribución de Hispanoamérica. Teoría y Praxis, 13, 9-33.

Chavoya, J., García J. y Pérez M. (2010). Puerto Vallarta: de ciudad turística a destino de segunda residencia. Procesos e implicaciones con el sector inmobiliario. En D. González 
(coord.), Puerto Vallarta en el imaginario. Procesos de su desarrollo (pp. 69-84). México: Universidad de Guadalajara.

Clancy, M (2001). Mexican tourism: export growth and structural change since 1970. Latin American Research Review, 36, 128-150.

De Kadt, E. (1984). Tourism: Passport to Development? Perspectives on the social and cultural effects of tourism in developing countries. Washington: Oxford University Press.

Díaz, P. (2013). Transformación y urbanización del frente costero español. En R. Pié i Ninot, C. J. Rosa Jiménez (Eds.), Turismo líquido (pp. 192-205). Barcelona: Instituto Hábitat Turismo Territorio a través de Iniciativa Digital Politècnica (UPC).

Everitt, J, Ryan H., Chávez, R. Espinosa R y Andrade E. (2008). The imprints of tourism on Puerto Vallarta, Jalisco, Mexico, Le Geographe Candien, 52(1), 83-104.

García, A. (1979). Cancún: Turismo y Subdesarrollo Regional. México: Universidad Nacional Autónoma de México.

Garibay, R. (1979). Acapulco. México: Editorial Grijalbo.

Garza G., Filion P. y Sands G. (2003). Políticas urbanas en grandes metrópolis: Detroit, Monterrey y Toronto. México: Centro de Estudios Demográficos y de Desarrollo Urbano, Programa Interinstitucional de Estudios sobre la Región de América del Norte.

Gómez, S. (2003). Movimientos sociales y sustentabilidad del turismo: los casos de dos conflictos en el destino turístico de Puerto Vallarta. En S. Gómez (coord.), Desarrollo Turístico y Sustentabilidad (103-134). México: Universidad de Guadalajara.

Gómez, S. (2012). La planificación de los destinos turísticos mexicanos: una receta mil veces vanagloriada". Investigaciones Turísticas, 4, 77-98.

Gómezjara, F. (1974). Acapulco: despojo y turismo". Problemas del Desarrollo. UNAM, 5(19), 127-147.

Graizbord, B. (1999). Planeación urbana, participación ciudadana y cambio social. Economía, Sociedad y Territorio, vol. II, núm. 5, 149-161.

Hiernaux, D. (1991). Cancun bliss. Dennis R. and S. Fainstein. The Tourist City. Yale University Press, New Haven and London.

Hiernaux, D. (2005). La promoción inmobiliaria y el turismo residencial: el caso mexicano. Scripta Nova, 9(194).

Hiernaux, D. (2010). Acapulco: nuevos espacios y estilos de vida. En D. Hiernaux (coord.), Las segundas residencias en México: un balance (pp. 99-117). México: Plaza y Valdés Editores y Universidad del Caribe, Colección Geografía.

Hiernaux, D. y Rodríguez, M. (1991). Las ciudades del turismo. Revista Mexicana de Ciencias Políticas y Sociales, 34(145), 13-25.

Jiménez, A. (1992). Turismo. Estructura y desarrollo. La estructura funcional del turismo internacional y la política turística de México. Desarrollo histórico. 1945-1990. México: McGraw-Hill.

Jiménez, A., y Sosa P. (2007). Desarrollo del turismo, impactos sociales y la planeación de ciudades turísticas: el caso de Cancún. En C. Silva y A. Iracheta (coord.), El futuro de las ciudades y el turismo. Memorias del VIII Seminario-Taller Internacional de la red mexicana de ciudades hacia la sustentabilidad (pp. 221-243). Quintana Roo: Instituto de Planeación de Desarrollo Urbano.

Marín, G (2015). Sin tierras no hay paraíso. Turismo, organizaciones agrarias y apropiación territorial en México. España: Pasos Revista de Turismo y Patrimonio Cultural.

Marín, G. (2008). Territorios de resistencia, integración mercantil y producción del espacio turístico en Quintana Roo: trayectorias y transformaciones del mundo maya. En A. 
Castellanos y J. Machuca (comps.), Turismo, identidades y exclusión (189-211). México: Universidad Autónoma Metropolitana, Unidad Iztapalapa y Casa Juan Pablos.

Mullins, P. (1991). Tourism urbanization, International Journal of Urban and Regional Science, 15(3), 326-342.

Munguía, C. (1997). Panorama histórico de Puerto Vallarta y de la Bahía de Banderas. México, Secretaría de Cultura de Gobierno de Jalisco/Ayuntamiento de Puerto Vallarta.

Núñez P. y Scartascini G. (2010). Política económica y desarrollo regional. En P. Núñez y S. Arnaiz. (coords.), Impactos y dimensiones del turismo (pp. 65-86). Puerto Vallarta: Universidad de Guadalajara.

Oehmichen, C. (2010). Cancún: la polarización social como paradigma en un México Resort. Revista Alteridades, México, 20(40).

Oehmichen, C. (2013). Una mirada antropológica al fenómeno del turismo. En C. Oehmichen (Edit.), Enfoques antropológicos sobre el turismo contemporáneo (pp. 35-72). México: UAM, Instituto de Investigaciones Antropológicas.

Olivares, A. (2014). Habitar las regiones urbanas turísticas. Seis formas de domesticar el espacio en la Región Puerto Vallarta-Bahía de Banderas. Arquitectura, Ciudad y Entorno, 9(25), 525-549.

Olveda J. (2011). La costa de la Nueva Galicia. Conquista y Colonización. Zapopan: El Colegio de Jalisco.

Oteiza, T. (1973). Acapulco. La ciudad de las Naos de Oriente y de las sirenas modernas. México: Editorial Diana.

Pié R. i Ninot, C. y Rosa C. (2013). Un turismo sin arquitectura o una arquitectura sin argumento. En R. Pié i Ninot, C. J. Rosa Jiménez (Eds.), Turismo líquido (pp. 6-11). Barcelona: Instituto Hábitat Turismo Territorio a través de Iniciativa Digital Politècnica (UPC).

Ramírez I., Osorio M., Cortés I. (2015). Tendencias y problemas actuales relacionados con la actividad turística. En D. Hiernaux (coord.), Turismo, Sociedad y Territorio: Una lectura crítica (pp. 121-146). México: Universidad Autónoma de Querétaro.

Ramírez J. (1986). Turismo y medio ambiente: El caso de Acapulco. Revista de Estudios Demográficos y Urbanos, 479-512.

Ramírez, M. (2009). El desarrollo urbano en Acapulco la normatividad para su ordenamiento y sus efectos en la zona Diamante (Tesis de maestría). Instituto Politécnico de Acapulco, Escuela Superior en Ingeniería y Arquitectura.

Rosa, C. (2013). El turismo como futuro: la ciudad del ocio. Turismo líquido. En R. Pié i Ninot, y C. J. Rosa Jiménez (Eds.), Turismo líquido (pp. 40-49). Barcelona: Instituto Hábitat Turismo Territorio a través de Iniciativa Digital Politècnica (UPC).

Santamaría, Arturo (2002). Los años dorados del turismo en Mazatlán. Mazatlán: Horson Ediciones Escolares.

SEDESOL-CONAPO-INEGI 2005. Delimitación de las zonas metropolitanas de México 2004. Aguascalientes. Sedesol-Conapo-INEGI.

Torres R. y Momsen J. (2005). Gringolandia: The Costruction of a New Tourist Space in Mexico. Annals of the Association of American Geographers, 95(2), 314-355.

Toscana, A. (2003). Impacto del huracán Paulina en la política local de Acapulco. Política y cultura, México, 19, 65-79.

Valenzuela E. y Coll A. (2010). La construcción y evolución del espacio turístico de Acapulco (México). Anales de Geografía, 30(1), 163-190. 
Vásquez, P. (2010). La planeación, el presupuesto y las políticas públicas. En M. Ordorica y J. Prud'homme (coord.), Los grandes problemas de México (pp. 106-141). México: El Colegio de México, Vol. XIII.

Véloz, C. (s/f). La planeación urbana en Cancún. México: Recuperado de http:yampu.com.

Verduzco, B. (2013). Una utopía urba (im) posible. La negociación infinita de planes de desarrollo urbano y prosperidad. Guadalajara: Universidad de Guadalajara.

Wong, P. (2009). Ordenamiento ecológico y ordenamiento territorial: retos para la gestión del desarrollo regional sustentable en el siglo XXI. Estudios Sociales, 17.

Para citar este artículo: Cárdenas, E.P. (2016). Crecimiento y planeación urbana en Acapulco, Cancún y Puerto Vallarta (México). Investigaciones Turísticas, (12), 99-120. http://dx.doi.org/10.14198/INTURI2016.12.05 